\title{
Molecular markers based on SNPs in FAD3 genes for determination of linolenic acid content in flax seed
}

\author{
Liubov Povkhova \\ Engelhardt Institute of Molecular \\ Biology, RAS, Moscow, Russia; \\ Moscow Institute of Physics and \\ Technology, Dolgoprudny, Russia \\ povhova.lv@phystech.edu \\ Elena Pushkova \\ Engelhardt Institute of Molecular \\ Biology, RAS, Moscow, Russia \\ pushkova18@gmail.com \\ Alexey Dmitriev \\ Engelhardt Institute of Molecular \\ Biology, RAS, Moscow, Russia \\ alex_245@mail.ru
}

\author{
Parfe Kezimana \\ Engelhardt Institute of Molecular \\ Biology, RAS, Moscow, Russia; \\ RUDN University, Moscow, Russia \\ k1par@mail.ru
}

Roman Novakovskiy

Engelhardt Institute of Molecular

Biology, RAS, Moscow, Russia

0legovich46@mail.ru

Nataliya Melnikova

Engelhardt Institute of Molecular

Biology, RAS, Moscow, Russia

mnv-4529264@yandex.ru
Tatiana Rozhmina

Engelhardt Institute of Molecular

Biology, RAS, Moscow, Russia;

Federal Research Center for Bast Fiber

Crops, Torzhok, Russia

tatyana_rozhmina@mail.ru

George Krasnov

Engelhardt Institute of Molecular

Biology, RAS, Moscow, Russia

gskrasnov@mail.ru

\begin{abstract}
Flax is grown for seeds in many countries. Depending on the ratio of fatty acids, seeds are used in pharmaceutical, food, feed, or polymer production. It is known that genes of $F A D 3$ family responsible for the desaturation of linoleic (LIO) into linolenic (LIN) fatty acid and some alleles of these genes are associated with a certain composition of flax oil. However, the development of the marker-assisted selection of flax is complicated due to a lack of methods for the identification of these polymorphisms. In the present study, we used 84 flax cultivars/lines with diverse content of LIO and LIN to search for fatty acid associated SNPs and then developed HRM-based system for identification of these SNPs that can increase the efficiency of breeding by obtaining pure improved cultivars with the targeted LIO and LIN content.
\end{abstract}

Keywords - Flax, fatty acids, FAD, LIN, LIO

Motivation and aim

Motivation

Flax (Linum usitatissimum L.) is grown for seeds in many countries. Linolenic (LIN), linoleic (LIO), and oleic (OLE) fatty acids are the main in flax oil, and depending on the ratio of these acids, seeds are used in pharmaceutical, food, feed, or polymer production. Flax genotypes differ in oil composition, and the breeding of cultivars with the particular content of LIN, LIO, and OLE is necessary. It is known that genes of $F A D$ (fatty acid desaturase) family play a key role in the synthesis of fatty acids. FAD3 genes are responsible for the desaturation of LIO into LIN, and some alleles of these genes are associated with a certain composition of flax oil [1]. However, the development of the marker-assisted selection of flax is complicated due to a lack of methods for the identification of DNA polymorphisms affecting the fatty acid content in flax seeds. Besides, cultivar heterogeneity could result in the presence of genotypes with different alleles of $F A D$ genes and diverse oil composition in individual flax cultivar, and molecular markers for these genes can be useful to increase the cultivar purity.
Aim

The purpose of our study was to develop a test system for the identification of SNPs (single nucleotide polymorphisms) associated with the fatty acid composition of flax oil.

\section{Methods}

For the present study, we used 84 flax cultivars/lines with diverse content of LIO and LIN in oil. Seeds were obtained from the Institute for Flax (Torzhok, Russia). DNA was extracted from flax seedlings using a CTAB protocol. For identification of SNPs, which are associated with fatty acid composition, deep sequencing of FAD $3 A$ and $F A D 3 B$ was performed on MiSeq (Illumina, USA) as described earlier [2]. VarScan [3] and freeBayes [4] variant callers were used for the identification of polymorphisms in the studied genes. Spearman's and Kendall's rank correlation coefficients between the content of LIO and LIN and polymorphisms in $F A D 3 A$ and $F A D 3 B$ genes were calculated. For identification of SNPs that were associated with oil composition, primers for the HRM (high-resolution melting) analysis were designed and PCR (polymerase chain reaction) conditions were optimized. HRM was performed on a Rotor-Gene Q real-time PCR cycler (Qiagen, Netherlands). Reaction mix contained 1x Taq Turbo buffer (Evrogen, Russia), $400 \mathrm{nM}$ of each primer (Evrogen), $250 \mu \mathrm{M}$ dNTPs (Evrogen), 1x EvaGreen (Biotium, USA), 1U of HS Taq DNA polymerase (Evrogen), and $40 \mathrm{ng}$ of DNA. The following PCR program was used: $95^{\circ} \mathrm{C}$ for 10 min followed by 40 cycles of $95^{\circ} \mathrm{C}$ for $15 \mathrm{~s}$ and $60^{\circ} \mathrm{C}$ for $60 \mathrm{~s}$. All reactions were performed in three technical replicates. Then, HRM analysis with the ramp from $65^{\circ} \mathrm{C}$ to $85^{\circ} \mathrm{C}$ rising by $0.1^{\circ} \mathrm{C}$ each step was conducted. For data analysis, RotorGene HRM software was used.

\section{Results}

In the present study, deep sequencing on the Illumina platform was used to identify polymorphisms in FAD3 genes in the representative set of 84 flax cultivars and lines. As a result, $300+300-b p$ reads with an average coverage of $300 x$ enabled accurate assessment of SNPs in FAD3A and FAD3B genes taking into account cultivar and line heterogeneity. For 
$F A D 3 A$ and $F A D 3 B$ genes, 91 and 62 polymorphisms were identified, respectively. Correlation analysis revealed polymorphisms in FAD3 genes that were associated with the ratio of fatty acids in flax oil. All except one of the samples with low LIN and high LIO had both the tryptophan to stop codon substitution in FAD $3 A$ and the histidine to tyrosine substitution in $F A D 3 B$, while all cultivars and lines with high LIN and low LIO had not these mutations. Samples with only one of the two mutations had medium content of LIN and LIO. The rest low-LIN sample contained another mutation in FAD3A resulting in arginine to stop codon substitution that was inherent only to this cultivar from the analyzed collection. Based on data on these three polymorphisms of FAD3 genes, controlling the LIN/LIO ratio, an HRM-based test system for the determination of linolenic acid content in flax seed was developed. Primers specific to conservative regions were designed taking into account all polymorphisms identified by us and other researchers. The HRM analysis with the preamplification method was used to get melt curves for the samples that were used for deep sequencing. Temperature shift in sample melt curves for three analyzed SNPs in homozygous and heterozygous states was distinguished clearly that enabled screening of flax for identification of associated with LIO and LIN content polymorphisms.
Molecular markers for the fatty acid composition of flax oil can increase the efficiency of breeding by obtaining pure improved cultivars and are necessary for the development of the marker-assisted selection of flax cultivars with the targeted LIO and LIN content.

\section{Acknowledgment}

This work was funded by RFBR according to the research project 17-29-08036.

\section{References}

[1] Thambugala D., et al. (2013) Genetic variation of six desaturase genes in flax and their impact on fatty acid composition. TAG Theoretical and applied genetics Theoretische und angewandte Genetik. 126(10): 2627-2641.

[2] Melnikova N.V., et al. (2019) Sex-specific polymorphism of MET1 and ARR17 genes in Populus x sibirica. Biochimie. 162: 26-32.

[3] Koboldt D.C., et al. (2012) VarScan 2: somatic mutation and copy number alteration discovery in cancer by exome sequencing. Genome Res. 22(3): 568-576.

[4] Garrison E., Marth G. (2012) Haplotype-based variant detection from short-read sequencing. arXiv:1207.3907v2 [q-bio.GN]. 